\title{
Identificação de Intervenções Tutoriais para Ambientes Virtuais de Aprendizagem
}

\author{
Soelaine Rodrigues Ascari ${ }^{1,2}$, Ernani Gottardo ${ }^{3}$, Andrey Ricardo Pimentel ${ }^{1}$ \\ ${ }^{1}$ Programa de Pós-graduação - Universidade Federal do Paraná (UFPR) \\ Curitiba - PR - Brasil \\ ${ }^{2}$ Departamento de Informática - Universidade Tecnológica Federal do Paraná (UTFPR) \\ Pato Branco - PR - Brasil \\ ${ }^{3}$ Instituto Federal de Educação, Ciência e Tecnologia do RS (IFRS) \\ Erechim - RS - Brasil \\ soelainedutfpr.edu.br, ernani.gottardoderechim.ifrs.edu.br, \\ andrey@inf.ufpr.br
}

\begin{abstract}
The objective of this work is to present which tutorial interventions, based on the classification of the type of error made by the learner, most contributed to a correct solution of exercises. For this purpose, an experiment using the game of mathematical fractions was performed in a real environment. In the game, a tutorial intervention model was implemented which, in addition to indicate the intervention based on error, performs the inference of the learner's emotions allowing to follow his changes in affective states. In this context, the results indicate that the interventions of the type Tips/Conveyed Information and the type Feedback/Explanatory and Goal or objective as the ones that most helped the apprentices to correctly answer the operations.
\end{abstract}

Resumo. O objetivo desde trabalho é apresentar quais intervenções tutoriais, a partir da classificação do tipo de erro cometido pelo aprendiz, mais contribuíram para uma solução correta dos exercícios. Para isso, um experimento utilizando o jogo de frações matemáticas foi realizado em um ambiente real. No jogo foi implementado um modelo de intervenção tutorial que além da indicação da intervenção com base no erro, realiza a inferência das emoções do aprendiz permitindo acompanhar suas mudanças de estados afetivos. Nesse contexto, os resultados indicam que as intervenções do tipo Dicas/Informação transmitida e o tipo Feedback/Explicativo e Meta ou de objetivo foram os que mais auxiliaram os aprendizes a responder corretamente as operações.

\section{Introdução}

O erro cometido pelo aprendiz, seja por falta de conhecimento de conceitos ou mesmo por desatenção, não precisa ser visto como algo negativo no processo de aprendizagem [Marczal et al. 2015]. O erro no campo matemático é considerando um evento natural, comum na trajetória do aprendiz que independe da idade e/ou do nível de desempenho [Leite et al. 2012]. Identificar esses erros pode ajudar o aprendiz a compreender seus erros para que possa corrigi-los em seguida. Com base nos erros, os Sistemas Tutores Inteligentes (STIs) têm a capacidade de identificar de forma individualizada as dificuldades 
IX Congresso Brasileiro de Informática na Educação (CBIE 2020)

Anais do XXXI Simpósio Brasileiro de Informática na Educação (SBIE 2020)

do aprendiz e assim prover uma intervenção tutorial mais apropriada [Leite et al. 2012] e [Marczal et al. 2015].

A intervenção tutorial tem um papel importante nos STIs, possibilitando manter o controle da situação do tutorial, para resguardar o aprendiz de uma aprendizagem incorreta ou inadequada, além de impedir que o aprendiz explore caminhos (objetivos instrucionais) que não sejam instrucionalmente úteis [Burns and Capps 2013]. Para isso, ao automatizar o processo de intervenção tutorial deve-se planejar e definir o momento que o aprendiz será interrompido, o tipo de intervenção tutorial e de que forma o conteúdo da intervenção será apresentada. Quanto ao momento da intervenção, para [Vanlehn 2006], o comportamento de um STI pode ser composto por dois laços: um laço externo (outer loop) que é executado uma vez por tarefa, e um laço interno (inner loop) que é executado uma vez para cada etapa. O foco desse trabalho é nas intervenções de laço interno.

O objetivo deste estudo é apresentar quais intervenções tutoriais, a partir da identificação e classificação do tipo de erro cometido pelo aprendiz, são mais adequadas ou que mais auxiliam os aprendizes na resolução correta dos exercícios. Assim, um modelo de intervenção tutorial foi desenvolvido e implementado em um jogo de frações matemáticas. O modelo utiliza a classificação de erros matemáticos de [Leite et al. 2012] para identificar os erros cometidos pelos aprendizes e para a indicação das intervenções tutoriais mais adequadas de forma a propiciar um maior engajamento e motivação. Além disso, é realizada a inferência do estado afetivo do aprendiz utilizando a abordagem de representação das emoções relacionadas à aprendizagem em quadrantes de [Gottardo and Pimentel 2018]. Um experimento foi realizado em ambiente real e os resultados indicam neste contexto quais as intervenções que mais auxiliam os aprendizes e propiciaram motivação e engajamento.

\section{Classificação de erros}

Estudos como os de [Movshovitz-Hadar et al. 1987], [Peng and Luo 2009] e [Leite et al. 2012] apresentam modelos ou teorias sobre classificação de erros matemáticos. Para [Leite et al. 2012] a necessidade de conhecimentos específicos do conteúdo a ser abordado e as causas que originam a situação de erro, é um grande desafio para o diagnóstico de erros matemáticos. E observa-se ainda que a diversidade e a complexidade desses erros tornam a tarefa de classificação de erros mais trabalhoso. Neste sentido, [Leite et al. 2012] propôs uma nova classificação de erros matemáticos com os seguintes tipos de erros:

- Interpretação equivocada: esse tipo de erro alertaria para a dificuldade do aprendiz em avançar na compreensão da estrutura do problema, ou seja, não consegue avançar em nenhuma estratégia porque pode estar com dificuldade de passar o problema da linguagem verbal para a matemática.

- Diretamente identificável: esse tipo é subclassificado em erros de deficiência em relação ao domínio ou uso inadequado de dados, erros de deficiência de regra, teorema ou definição e erros de deficiência na escolha do operador correto.

- Indiretamente identificáveis: esse tipo contempla o erro apresentado pela falta de lógica correta.

- Solução não categorizável: o erro que não esteja compreendido em nenhum dos tipos acima será incluído nessa classificação. 
IX Congresso Brasileiro de Informática na Educação (CBIE 2020)

Anais do XXXI Simpósio Brasileiro de Informática na Educação (SBIE 2020)

\section{Intervenções Tutoriais}

Esta seção apresentada um estudo sobre os tipos de intervenções tutoriais utilizados nos ambientes virtuais de aprendizagem. Foi criada uma classificação a partir dos trabalhos de [McKendree 1990], [Fleming and Levi 1993], [Hume et al. 1996], [Hannafin et al. 1999], [McLoughlin 2004], [Economides 2005] e [Narciss 2013]. O primeiro tipo é o Scaffolding, esse termo é utilizado como uma metáfora no processo de aprendizagem, por referir-se ao apoio temporário que é oferecido ao aprendiz para o desenvolvimento de uma atividade. Em seguida tem-se o Feedback, que são todas as informações pós-resposta que informam os aprendizes sobre seu estado real de aprendizado ou desempenho, além de propiciar ajuda ao aprendiz de modo que ele tenha condições de solucionar uma tarefa e com isso progredir no aprendizado. E por fim as Dicas ou sugestões (tips or hints), buscam lembrar os aprendizes das informações que se presume serem de seu conhecimento, ou seja, estimular a recordação de fatos que os auxiliem a responder a uma pergunta. A Tabela 1 apresenta os tipos e seus respectivos subtipos de intervenções.

Tabela 1. Tipos e subtipos de intervenções.

\begin{tabular}{|c|c|c|}
\hline Tipo & Subtipo & Referência \\
\hline Scaffolding & Conceitual, Processual, Estratégico e Metacognitivo. & $\begin{array}{l}{[\text { McLoughlin2004] }} \\
\text { [Hannafin et al. } \\
\text { 1999] }\end{array}$ \\
\hline & $\begin{array}{l}\text { Baseado na função: Feedback de Confirmação, Corretivo, } \\
\text { Explicativo, Diagnóstico e Elaborativo. }\end{array}$ & $\begin{array}{l}\text { [Fleming and Levi } \\
\text { 1993] }\end{array}$ \\
\hline Feedback & $\begin{array}{l}\text { Baseado no conteúdo: Conhecimento da resposta, Resposta até } \\
\text { que seja correta, Tópico contingente, Resposta contingente, } \\
\text { Bug relacionado, Isolamento de atributos, Conhecimento do } \\
\text { desempenho, Conhecimento da resposta correta, Elaborado, de } \\
\text { violacão de condicão, de meta ou de objetivo e Combinado. }\end{array}$ & $\begin{array}{l}{[\text { McKendree 1990] }} \\
{[\text { Economides 2005] }} \\
{[\text { Narciss 2013] }}\end{array}$ \\
\hline Dicas & $\begin{array}{l}\text { Informação transmitida (IC - Conveyed Information), Apontar } \\
\text { para (PT - Pointing To) e Linha direta de raciocínio (DLR - } \\
\text { Directed Line of Reasoning). }\end{array}$ & [Hume et al. 1996] \\
\hline
\end{tabular}

Dos 24 subtipos de intervenções apresentados na Tabela 1, seis foram selecionados para serem apresentados aos aprendizes sempre que uma resposta não estava correta. A escolha dos subtipos levou em consideração o conteúdo (operações sobre frações), a forma como ele é apresentado e em função de adequação ao modelo do jogo. A Tabela 2 apresenta os subtipos que foram implementados.

\section{Trabalhos relacionados}

Foram encontrados na literatura alguns trabalhos que abordam o tema deste artigo, entre eles, destacam-se os seguintes: 1) O trabalho de [Leite et al. 2012] apresenta uma arquitetura para remediação de erros baseada nas teorias de classificações de erros matemáticos, das Múltiplas Representações Externas (MREs) e teoria Adaptive Control of Thought (ACT). Neste trabalho o erro do aprendiz é vinculado a função do MRE adequada ao erro cometido, identifica a MRE e realiza a remediação. 2) Outro trabalho de [Leite et al. 2015] prove remediações de erro mais adequadas a cada aprendiz ao contexto de interação. 3) A pesquisa de [Marczal et al. 2016] apresenta uma ferramenta de autoria para Objetos de Aprendizagem, que permite a manipulação dos registros de erros por 
IX Congresso Brasileiro de Informática na Educação (CBIE 2020)

Anais do XXXI Simpósio Brasileiro de Informática na Educação (SBIE 2020)

Tabela 2. Tipos e subtipos de intervenções implementadas.

\begin{tabular}{|l|l|}
\hline \multicolumn{1}{|c|}{$\begin{array}{c}\text { Tipo/Subtipo de } \\
\text { intervenção }\end{array}$} & \multicolumn{1}{|c|}{ Quando usar } \\
\hline Feedback Explicativo & $\begin{array}{l}\text { Apresentar informações relevantes que ajude a identificar por que uma } \\
\text { resposta está incorreta. }\end{array}$ \\
\hline Feedback Diagnóstico & Para evidenciar o erro e indicar uma solução. \\
\hline $\begin{array}{l}\text { Feedback de meta ou } \\
\text { de objetivo }\end{array}$ & $\begin{array}{l}\text { Fornecer estímulo ou uma sugestão do caminho que precisa ser seguido } \\
\text { para encontrar a solução correta. }\end{array}$ \\
\hline $\begin{array}{l}\text { Dica Informação } \\
\text { transmitida }\end{array}$ & $\begin{array}{l}\text { Solicitar ao aprendiz que infira ou recorde de uma resposta, ou ainda da } \\
\text { próxima etapa de uma solução. }\end{array}$ \\
\hline Dica Apontar para & $\begin{array}{l}\text { Apontar para a localização da informação em uma base de } \\
\text { conhecimento, por exemplo, mas sem transmitir a informação. }\end{array}$ \\
\hline $\begin{array}{l}\text { Dica Linha direta de } \\
\text { raciocínio }\end{array}$ & $\begin{array}{l}\text { Quando deseja-se fazer com que o aprendiz tenha que pensar sobre cada } \\
\text { uma das etapas até chegar em uma solução. }\end{array}$ \\
\hline
\end{tabular}

meio de um mecanismo que possibilita remediação e retroação a erros a curto e longo prazo. 4) No trabalho de [Gottardo and Pimentel 2018] é apresentada uma classificação de eventos monitorados, entre eles acertos e erros nas questões. Após a ocorrência do evento é feita inferência do estado afetivo do aprendiz e o mapeamento de acordo com a representação das emoções em quadrantes.

Verificou-se que os trabalhos correlatos apresentam intervenções, baseados nos erros do aprendiz, em forma de MRE ou de mensagens de texto. Este trabalho apresenta a intervenção tutorial com base em uma classificação de intervenções tutorias de acordo com a identificação e classificação do tipo de erro cometido pelo aprendiz. Além disso, é realizada a inferência do estado afetivo do aprendiz utilizando a abordagem de representação das emoções em quadrantes [Gottardo and Pimentel 2018], baseado nas emoções básicas do modelo Ekman [Ekman 1992]. Desse modo é possível verificar quais intervenções mais auxiliaram os aprendizes na resolução dos exercícios de forma a propiciar um maior engajamento e motivação.

\section{Experimento}

Esta seção apresenta as informações referentes ao experimento com o jogo de frações aplicado em um ambiente real. Participaram do experimento 34 aprendizes de duas Escolas Municipais, do quinto ano do ensino fundamental com idades entre 10 e 12 anos, sendo 10 do sexo feminino e 24 do sexo masculino. O experimento foi realizado nos laboratórios de informática das escolas entre os meses de novembro e dezembro de 2019. Este experimento foi aprovado e cumpriu com todos os procedimentos recomendados pelo Comitê de Ética em Pesquisa em Seres Humanos da UFPR (CEP/SD).

Nos dias de aplicação dos experimentos os laboratórios eram preparados de modo que os aprendizes ao chegarem encontravam os computadores ligados, com o jogo carregado e webcams configuradas. Antes de iniciar o experimento os aprendizes receberam informações sobre o objetivo e funcionamento do jogo, assim como lembrados que as webcams estariam gravando sua imagem, mas que essas não seriam divulgadas. As turmas precisaram ser divididas em função do número de computadores, assim, foram realizadas duas sessões com cada turma de cada escola com tempo médio de 45 minutos. Cabe ressaltar que todos os aprendizes concluíram o experimento. 
IX Congresso Brasileiro de Informática na Educação (CBIE 2020)

Anais do XXXI Simpósio Brasileiro de Informática na Educação (SBIE 2020)

\subsection{Modelo de intervenção}

A Figura 1 é uma representação do modelo de intervenção tutorial que indica intervenções tutoriais a partir da identificação do tipo de erro do aprendiz, além de inferir o estado afetivo.

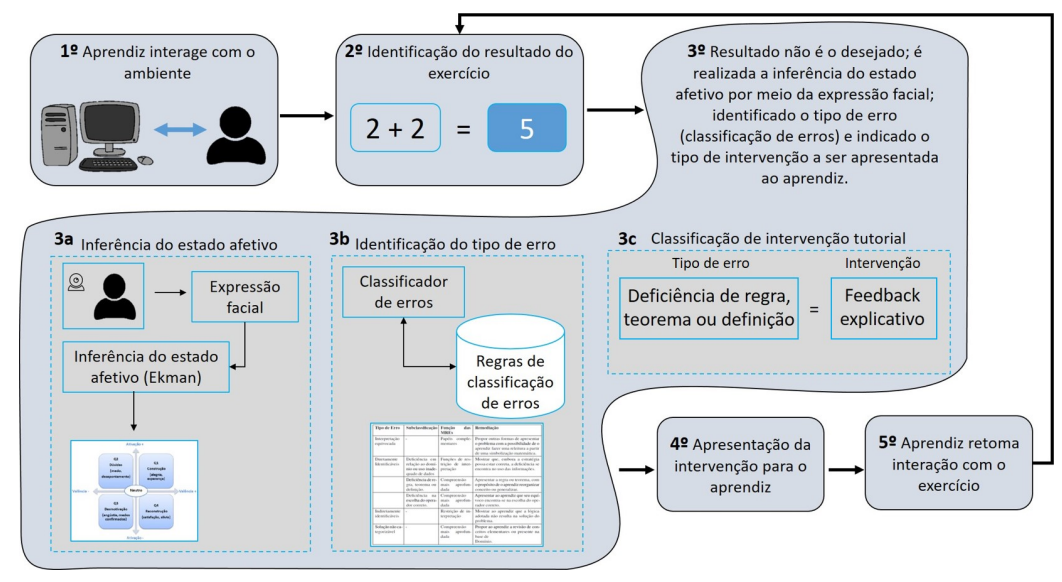

Figura 1. Modelo de inervenção tutorial.

O aprendiz interage com o ambiente, neste caso o jogo de frações, e ao responder o exercício $\left(1^{\circ}\right)$ o modelo identifica se a resposta está certa ou não $\left(2^{\circ}\right)$. Se a resposta estiver correta o ambiente carrega um novo exercício. Caso contrário, o ambiente identifica qual é o tipo de erro, e simultaneamente captura a imagem facial do aprendiz. Para a identificação do tipo de erro (3.a) foi utilizada a classificação de erros matemáticos, desenvolvida por [Leite et al. 2012] conforme apresentados na seção 2. Já a inferência do estado afetivo é realizada com base na expressão facial do aprendiz capturada por uma câmera padrão de acordo com as emoções básicas do modelo de Ekman [Ekman 1992]. O modelo de inferência aplicado utiliza a abordagem de representação das emoções em quadrantes, formados pelas dimensões valência (eixo horizontal) e ativação (eixo vertical) que foram nomeados como Q1, Q2, Q3, Q4, além de um estado Neutro (QN), conforme a Figura 2 [Gottardo and Pimentel 2018]. Os quadrantes agregam um conjunto de emoções que tem potencial de impactar o processo de aprendizagem. Os quadrantes Q1 e Q2 com ativação positiva formam a classe chamada agitação, e os quadrantes Q3 e Q4 com ativação negativa formam a classe sonolência.

Após detectar o tipo/subtipo de erro e inferir o estado afetivo (3c), uma intervenção tutorial é exibida para o aprendiz em forma de texto em linguagem natural. Após ler a intervenção ele poderá responder novamente o exercício, se a resposta estiver correta o ambiente carrega um novo exercício, caso contrário retorna à terceira etapa. Assim, o objetivo do modelo é apresentar para os aprendizes intervenções tutoriais que os auxiliem na resolução dos exercícios, além de mantê-los motivados.

\subsection{Jogo de frações}

O jogo de frações matemáticas foi desenvolvido em linguagem Python utilizando o framework Django com o banco de dados SQLite. Para a detecção da expressão facial foi usado a API Azure da Microsoft. O acesso ao jogo foi realizado por meio de um website hospedado em um servidor local. Um teste piloto foi realizado, antes da aplicação do 


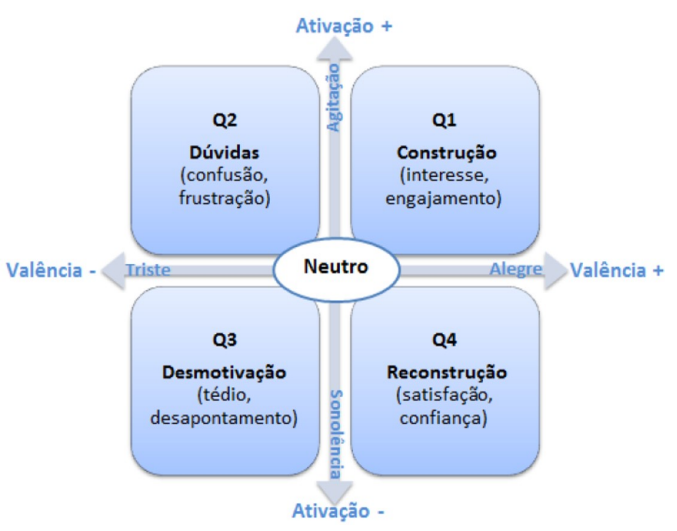

Figura 2. Modelo de representação das emoções em quadrantes.

Fonte: [Gottardo and Pimentel 2018].

experimento nas escolas, com a participação de três aprendizes da mesma faixa etária dos participantes do experimento. Assim, foi possível simular a aplicação do experimento, verificar o tempo para a resolução das operações, avaliar o registro dos dados e o funcionamento do jogo. Após a conclusão desta etapa adequações foram realizadas no jogo e na interface.

O jogo consiste na apresentação de 22 operações de frações que considera as quatro operações básicas: soma, subtração, multiplicação e divisão, essas divididas em quatro níveis de dificuldade. As operações foram avaliadas, ajustadas e aprovadas pelas professoras de matemática das turmas envolvidas com o experimento. Foram registrados os erros, os estados afetivos e as intervenções tutoriais aplicadas. Foi criado um avatar com a função de orientar e apresentar as intervenções para os aprendizes durante o jogo.

No jogo foram implementados os tipos e subtipos de acordo com os erros identificados em uma lista de exercícios impressa sobre frações matemáticas realizada por aprendizes do quinto ano do ensino fundamental. Com o auxílio de uma professora da área de matemática foi possível identificar e classificar a ocorrência dos seguintes tipos e subtipos de erros, considerando a classificação de erros matemáticos de Leite et al.(2012): 1) Diretamente identificável - erros de deficiência de regra, teorema e definição e erros de deficiência na escolha do operador correto; 2) Indiretamente identificáveis; e 3) Solução não categorizável. O tipo de erro interpretação equivocada e o subtipo deficiência em relação ao domínio ou uso inadequado de dados não foram identificados na lista de exercícios e dessa forma não foram implementados no jogo. A Tabela 3 apresenta uma operação do jogo com a indicação de um erro, seu tipo, subtipo, tipo e subtipo de intervenção.

Tabela 3. Operação com tipo/subtipo de erro e intervenção tutorial.

\begin{tabular}{|l|c|c|c|c|c|}
\hline Operação & $\begin{array}{c}\text { Resposta } \\
\text { incorreta }\end{array}$ & $\begin{array}{c}\text { Tipo do } \\
\text { erro }\end{array}$ & Subtipo do erro & $\begin{array}{c}\text { Tipo de } \\
\text { intervenção }\end{array}$ & $\begin{array}{c}\text { Subtipo de } \\
\text { intervenção }\end{array}$ \\
\hline$\frac{3}{5}+\frac{1}{5}=$ & $\frac{4}{10}$ & $\begin{array}{c}\text { Diretamente } \\
\text { identificável }\end{array}$ & $\begin{array}{c}\text { Defíciência de regra, } \\
\text { teorema ou definição }\end{array}$ & Feedback & Diagnóstico \\
\hline
\end{tabular}

A Tabela 4 apresenta os possíveis textos de intervenção que poderiam ser apresen- 
tados quando da soma de duas frações com denominadores iguais em que é verificado que o numerador do resultado está correto e o denominador não. Neste caso foi identificado o subtipo de erro "Deficiência de regra, teorema ou definição", pois o aprendiz aplicou parcialmente de forma correta a regra de soma de denominadores iguais.

Tabela 4. Textos de intervenções tutoriais.

\begin{tabular}{|c|c|c|c|c|c|l|}
\hline Tipo & $\begin{array}{c}\text { Subtipo de } \\
\text { Intervenção }\end{array}$ & OP & N & DS & D & \multicolumn{1}{|c|}{ Texto da intervenção } \\
\hline \multirow{2}{*}{ Dicas } & $\begin{array}{c}\text { Informação } \\
\text { transmitida }\end{array}$ & + & A & I & A & $\begin{array}{l}\text { Nas operações de soma com denominadores iguais deve-se } \\
\text { somar um dos termos e repetir o outro. }\end{array}$ \\
\cline { 2 - 6 } & $\begin{array}{c}\text { Linha direta } \\
\text { de raciocínio }\end{array}$ & + & A & I & A & $\begin{array}{l}\text { Os denominadores são iguais ou diferentes? Lembre-se que essa } \\
\text { informação define como resolver a operação de soma. }\end{array}$ \\
\hline Feedback & Diagnostico & + & C & I & N & $\begin{array}{l}\text { Resposta incorreta! O erro está no valor do denominador. Na } \\
\text { soma de frações com denominadores iguais basta repetir o } \\
\text { denominador no resultado. }\end{array}$ \\
\hline Explicativo & + & C & I & N & $\begin{array}{l}\text { Resposta incorreta, pois o denominador da resposta selecionada } \\
\text { não é o correto. Veja que é uma operação de soma e que os } \\
\text { denominadores são iguais. }\end{array}$ \\
\hline
\end{tabular}

A cada nova tentativa de resposta do aprendiz o jogo realizava um sorteio entre todas as intervenções que se enquadravam nas regras. Essas regras referem-se ao conjunto de informações coletadas da própria questão: OP (Operação), DS (Denominadores iguais ou diferentes), e ainda comparando os $\mathrm{N}$ (Numeradores) e D (Denominadores) da resposta selecionada com os da resposta correta. A OP pode ser uma soma (+), subtração (-), multiplicação (x) ou divisão (:). O DS verifica se os denominadores das frações são iguais (I) ou diferentes (D). O N corresponde ao numerador da fração, que pode estar correto $(\mathrm{C})$, não correto $(\mathrm{N})$ ou o valor não interfere $(\mathrm{A})$ na indicação da intervenção. Se após a terceira tentativa o aprendiz ainda não conseguiu responder corretamente a questão é exibida uma intervenção do tipo Feedback com a indicação da resposta correta e qual foi o erro cometido ou como ele deveria ter resolvido a questão.

\section{Resultados do Experimento e Discussões}

Foram registradas no total 1114 respostas (certas ou erradas), dessas 968 (88,5\%) são ocorrências identificadas, ou seja, que foi possível inferir o estado afetivo do aprendiz e mapear no quadrante correspondente, de acordo com a abordagem de representação de emoções por quadrante. Nas demais 128 respostas (11,5\%) não foi possível inferir o estado afetivo do aprendiz. Os principais motivos da não identificação foram: posição do aprendiz (fora do enquadramento da câmera) e mão na frente do rosto. A média de ocorrências por aprendiz foi de 32,8 com desvio padrão de 5,9.

Das ocorências identificadas o quadrante com o maior número de ocorrências de estados afetivos foi o QN com 889 (90,2\%), a predominância do estado neutro era esperado para essa atividade e alinha-se com o resultado de outros trabalhos nessa área [D'Mello et al. 2007] e [Gottardo and Pimentel 2018]. Na sequência o Q1 com 49 (5,0\%) ocorrências de estados afetivos como enganjamento, alegria e motivação, que são desejados para o processo de aprendizagem. O quadrante Q2 registrou $31(3,1 \%)$ ocorrências de emoções como medo, frustração e confusão. Esse resultado ajuda a confirmar que esses estados afetivos estão presentes em atividades de aprendizagem, podendo estar correlacionado positivamente com a aprendizagem [Pour et al. 2010] e [D'Mello et al. 2007]. Em seguida o quadrante Q4 com $14(1,4 \%)$ das ocorrências e por fim o Q3 com $3(0,3 \%)$ ocorrências. 
As intervenções tutoriais geraram 14 mudanças de algum quadrante para Q1, além de manter seis vezes o quadrante Q1 após a intervenção. Dessas houve cinco ocorrências de mudanças de quadrantes diferentes para Q1 a partir de intervenções que pertencem ao tipo Feedback e duas ocorrências de intervenções em que o aprendiz se manteve em Q1. Ainda foram registradas nove ocorrências de mudanças de quadrantes diferentes para Q1 a partir de intervenções do tipo Dicas e quatro ocorrências de intervenções onde o aprendiz se manteve em Q1. A partir desse resultado é possível concluir que as intervenções dentro do contexto deste trabalho conseguiu proporcionar motivação e engajamento.

A Tabela 5 apresenta quais intervenções tutoriais foram apresentadas para os aprendizes quando da identificação dos tipos/subtipos dos erros.

Tabela 5. Tipos e subtipos de erros $\mathrm{x}$ subtipos de intervenção.

\begin{tabular}{|l|l|c|c|c|c|c|c|c|}
\hline & Acertos & Erros & Total & $\begin{array}{c}\% \\
\text { Acerto }\end{array}$ & $\begin{array}{c}\% \\
\text { Erro }\end{array}$ & $\begin{array}{c}\text { Mudança } \\
\text { para Q1 }\end{array}$ & $\begin{array}{c}\text { Mudança } \\
\text { para Q2 }\end{array}$ \\
\hline Deficiência de regra, teorema ou definição & & & & & & & 0 \\
\hline Dicas & Apontar para & 0 & 1 & 1 & $0,0 \%$ & $100,0 \%$ & 0 & 0 \\
\hline Dicas & Informação transmitida & 24 & 51 & 75 & $32,0 \%$ & $68,0 \%$ & 5 & 2 \\
\hline Dicas & Linha direta de raciocínio & 1 & 8 & 9 & $11,1 \%$ & $88,9 \%$ & 0 & 1 \\
\hline Feedback & Diagnóstico & 2 & 0 & 2 & $100,0 \%$ & $0,0 \%$ & 0 & 0 \\
\hline Feedback & Explicativo & 21 & 38 & 59 & $35,6 \%$ & $64,4 \%$ & 3 & 2 \\
\hline Feedback & Meta ou de objetivo & 11 & 21 & 32 & $34,4 \%$ & $65,6 \%$ & 1 & 1 \\
\hline Deficiência na escolha do operador correto & & & & & & & \\
\hline Dicas & Informação transmitida & 4 & 10 & 14 & $28,6 \%$ & $71,4 \%$ & 0 & 0 \\
\hline Dicas & Linha direta de raciocínio & 1 & 0 & 1 & $100,0 \%$ & $0,0 \%$ & 0 & 0 \\
\hline Feedback & Meta ou de objetivo & 4 & 4 & 8 & $50,0 \%$ & $50,0 \%$ & 0 & 0 \\
\hline Indiretamente identificáveis & & & & & & & \\
\hline Dicas & Informação transmitida & 1 & 1 & 2 & $50,0 \%$ & $50,0 \%$ & 0 & 0 \\
\hline Feedback & Meta ou de objetivo & 1 & 2 & 3 & $33,3 \%$ & $66,7 \%$ & 0 & 0 \\
\hline Solução não categorizável & & & & & & & 0 \\
\hline Dicas & Apontar para & 2 & 0 & 2 & $100,0 \%$ & $0,0 \%$ & 0 & 0 \\
\hline Dicas & Informação transmitida & 28 & 47 & 75 & $37,3 \%$ & $62,7 \%$ & 3 & 4 \\
\hline Dicas & Linha direta de raciocínio & 8 & 15 & 23 & $34,8 \%$ & $65,2 \%$ & 1 & 2 \\
\hline Feedback & Diagnóstico & 2 & 0 & 2 & $100,0 \%$ & $0,0 \%$ & 1 & 0 \\
\hline Feedback & Explicativo & 13 & 26 & 39 & $33,3 \%$ & $66,7 \%$ & 0 & 3 \\
\hline Feedback & Meta ou de objetivo & 9 & 10 & 19 & $47,4 \%$ & $52,6 \%$ & 0 & 2 \\
\hline Total & & 234 & 366 & & & 14 & 17 \\
\hline
\end{tabular}

Sobre o total de 366 ocorrência de intervenções, 45,4\% correspondem as intervenções Dicas/Informação transmitida, seguida de Feedback/Explicativo com 26,8\%, depois Feedback/Meta ou de objetivo com 16,9\%, Dicas/Linha direta de raciocínio com 9,0\%, Feedback/Diagnóstico com 1,1\% e Dicas/Apontar para com 0,8\%. Com relação as 132 ocorrências de acertos 43,2\% das intervenções são Dicas/Informação transmitida, em segundo lugar Feedback/Explicativo com 25,8\%, Feedback/Meta ou de objetivo com 18,9\%, Dicas/Linha direta de raciocínio com 7,6\%, Feedback/Diagnóstico com 3,0\% e Dicas/Apontar para com 1,5\%. Observa-se que as intervenções Dicas/Informação transmitida, Feedback/Explicativo e Feedback/Meta ou de objetivo recebidas pelos aprendizes durante a resolução das operações de frações obtiveram os maiores valores percentuais de acertos em relação ao total de acertos.

Pode-se observar na Tabela 5 que as intervenções Dica/Informação Transmitida, para os erros "Deficiência de regra, teorema e definição" e "Solução não categorizável", e Feedback/Explicativo, para o erro "Deficiência de regra, teorema e definição", tiveram mudanças de quadrante com mais motivação e engajamento (mudança para Q1) e per- 
centual de acerto maior que as demais intervenções. Outra informação são as mudanças para quadrante Q2, que nem sempre está relacionado ao erro de forma negativa. Do total de 17 ocorrências de mudança para Q2, nove são mudanças em que o aprendiz acertou a resposta. Lembrando que Q2 possui ativação positiva, assim o aprendiz pode não estar motivado, mas agitado por querer resolver a operação apesar da inferência de emoção de confusão ou dúvida. O desempenho em atividades educativas tem relação com o nível de ativação do aprendiz, sendo os melhores níveis de desempenho aqueles obtidos com níveis intermediários de ativação [Shen et al. 2007].

Com base no contexto desse artigo os resultados demonstram que as intervenções que mais auxiliaram os aprendizes a responder corretamente as operações foram o tipo Dicas com o subtipo Informação transmitida e o tipo Feedback com o subtipo Explicativo e Meta ou de objetivo para todos os tipos de erros identificados.

\section{Conclusão}

Este trabalho de pesquisa tem como objetivo apresentar intervenções tutoriais a partir da identificação e classificação do tipo de erro cometido pelo aprendiz de forma que essas intervenções possam auxiliá-los na resolução das operações de frações, além de propiciar um maior engajamento/motivação. Dessa forma, foi apresentado um experimento com aprendizes do quinto ano do ensino fundamental aplicando um jogo de frações matemáticas.

Nesse contexto, observa-se que as intervenções tutoriais associadas e apresentadas a partir da identificação dos tipos de erros contribuíram para uma maior ocorrência de mudança e permanência dos aprendizes de outros quadrantes para Q1 (valência e ativação positiva). Nesse quadrante são mapeados os estados afetivos que contribuem e apresentam potencial de impactar o processo de aprendizagem.

Estes resultados, mesmo considerando a limitação no número de aprendizes participantes, poderiam ser utilizados para a definição e implementação de intervenções tutorias em ambientes virtuais de aprendizagem considerando o erro cometido pelo aprendiz como algo natural, como suporte ao processo de aprendizagem, pois permite ao aprendiz realizar uma análise da situação e ao mesmo tempo uma revisão de conceitos/conteúdos. Uma recomendação para trabalhos futuros é o mapeamento de eventos do jogo para possibilitar a inferência dos estados afetivo cognitivo do aprendiz.

\section{Referências}

Burns, H. L. e Capps, C. G. (2013). Foundations of intelligent tutoring systems: An introduction. Foundations of intelligent tutoring systems, page 1.

D'Mello, S., Picard, R. W., e Graesser, A. (2007). Toward an affect-sensitive autotutor. IEEE Intelligent Systems, 22(4):53-61.

Economides, A. A. (2005). Adaptive feedback evaluation. In Proceedings of the 5th WSEAS International Conference on Distance Learning and Web Engineering.

Ekman, P. (1992). An argument for basic emotions. Cognition and Emotion, 6:169-200.

Fleming, M. L. e Levi, W. H. (1993). Instructional message design: Principles from the behavioral and cognitive sciences. Educational technology. 
IX Congresso Brasileiro de Informática na Educação (CBIE 2020)

Anais do XXXI Simpósio Brasileiro de Informática na Educação (SBIE 2020)

Gottardo, E. e Pimentel, A. (2018). Reconhecimento e adaptação à dinâmica de estados afetivos relacionados à aprendizagem. In Anais do XXIX Simpósio Brasileiro de Informática na Educação (SBIE 2018).

Hannafin, M., Land, S., e Oliver, K. (1999). Open learning environments: Foundations, methods, and models. Instructional-design theories and models: A new paradigm of instructional theory, 2:115-140.

Hume, G., Michael, J., Rovick, A., e Evens, M. (1996). Hinting as a tactic in one-on-one tutoring. Journal of the Learning Sciences, 5(1):23-47.

Leite, D. M., Marczal, D., Krynski, E., Pimentel, A. R., Direne, A., e Reinaldo, F. (2015). Otimizando o processo de ensino e aprendizagem com a arquitetura para desenvolvimento de objetos de aprendizagem - adoa. Simpósio Brasileiro de Informática na Educação - SBIE, 26(1):1002.

Leite, M. D., Pimentel, A. R., e Pietruchinski, M. H. (2012). Remediação de erros baseada em múltiplas representações externas e classificação de erros aplicada a objetos de aprendizagem inteligentes. In Simpósio Brasileiro de Informática na Educação - SBIE, volume 23 .

Marczal, D., Direne, A., Pimentel, A., e Krynski, E. M. (2015). FARMA: Uma ferramenta de autoria para objetos de aprendizagem de conceitos matemáticos. In Anais dos Workshops do IV Congresso Brasileiro de Informática na Educação (CBIE 2015).

Marczal, D., Direne, A. I., Pimentel, A. R., Maschio, E., e Borille, A. C. (2016). Metodologia e software educacional para a investigação e remediação de erros conceituais em matemática. Revista Brasileira de Informática na Educação, 24(02):77.

McKendree, J. (1990). Effective feedback content for tutoring complex skills. Human-Computer Interaction, 5(4):381-413.

McLoughlin, C. (2004). Achieving excellence in teaching through scaffolding learner competence. Seeking Educational Excellence, pages 9-10.

Movshovitz-Hadar, N., Zaslavsky, O., e Inbar, S. (1987). An empirical classification model for errors in high school mathematics. Journal for research in mathematics Education, pages 3-14.

Narciss, S. (2013). Designing and evaluating tutoring feedback strategies for digital learning environments on the basis of the interactive tutoring feedback model. Digital Education Review, 23:7-26.

Peng, A. e Luo, Z. (2009). A framework for examining mathematics teacher knowledge as used in error analysis. For the learning of mathematics, 29(3):22-25.

Pour, P. A., Hussain, M. S., AlZoubi, O., D’Mello, S., e Calvo, R. A. (2010). The impact of system feedback on learners' affective and physiological states. In International Conference on Intelligent Tutoring Systems, pages 264-273. Springer.

Shen, L., León, E., Callaghan, V., e Shen, R. (2007). Exploratory research on an affective e-learning model.

Vanlehn, K. (2006). The behavior of tutoring systems. International journal of artificial intelligence in education, 16(3):227-265. 